\title{
Shock and awe: unleashing the heat shock response to treat Huntington disease
}

\author{
Meredith E. Jackrel and James Shorter
}

Department of Biochemistry and Biophysics, Perelman School of Medicine, University of Pennsylvania, Philadelphia, Pennsylvania, USA.

\begin{abstract}
The heat shock response (HSR) is a highly conserved protective mechanism that enables cells to withstand diverse environmental stressors that disrupt protein homeostasis (proteostasis) and promote protein misfolding. It has been suggested that small-molecule drugs that elicit the HSR by activating the transcription factor heat shock factor $1 \mathrm{might}$ help mitigate protein misfolding and aggregation in several devastating neurodegenerative disorders, including Huntington disease (HD). In this issue of the JCI, Labbadia et al. use a brain-penetrant Hsp90 inhibitor, HSP990, to induce the HSR in mouse models of HD. Unexpectedly, they observed that HSP990 confers only transient amelioration of a subset of HD-related phenotypes, because alterations in chromatin architecture impair the HSR upon disease progression. These findings suggest that synergistic combination therapies that simultaneously unleash the HSR and prevent its impairment are likely to be needed to restore proteostasis in $\mathrm{HD}$.
\end{abstract}

Proteins must fold into intricate 3-dimensional structures to perform an extraordinary variety of functions $(1,2)$. A complex network of highly conserved molecular chaperones ensures successful protein folding $(1,2)$. Aberrant protein folding that overwhelms this chaperone network is closely linked to several devastating neurodegenerative disorders, including Huntington disease (HD), Parkinson disease, and Alzheimer disease (1-3). There are no treatments for these disorders, all of which are associated with the misfolding, oligomerization, and aggregation of specific proteins in the CNS (1-3). HD is a fatal, inherited, late-onset neurodegenerative disorder that afflicts approximately 1 individual per 10,000 worldwide (4). It is caused by a CAG triplet repeat expansion in exon 1 of the huntingtin (HTT) gene, which generates an expanded polyglutamine tract (approximately 40 residues or longer) in the mutant huntingtin protein (4). This polyglutamine tract expansion increases the propensity of huntingtin protein to form a heterogeneous array of misfolded species, ranging from toxic oligomers to diverse aggregated structures $(5,6)$. An increasing number of potential therapeutic strategies now focus on unleashing the natural defenses that

Conflict of interest: The authors have declared that no conflict of interest exists.

Citation for this article: J Clin Invest. 2011; 121(8):2972-2975. doi:10.1172/JCI59190. cells have evolved to counter the proteotoxic stress caused by potentially deleterious protein-misfolding events (1, 7-9). In this issue of the JCI, Labbadia and colleagues find that although one such approach ameliorates some disease phenotypes in a mouse model of HD, the benefits are only transient (10), which indicates that synergistic combination therapies are likely to be needed if such strategies are to make it to the clinic.

In response to proteotoxic stress, cells maintain protein homeostasis (proteostasis) by rapidly adjusting the concentrations of appropriate molecular chaperones $(1,2)$. They do this by rapidly activating ancient and highly conserved pathways, such as the heat shock response (HSR), which responds to stress in the cytoplasm, and the unfolded protein response (UPR), which responds to stress in the secretory pathway $(1,2)$. These pathways upregulate the requisite components of the chaperone network. Molecular chaperones can then buffer aberrant protein conformers either by returning them to their native form or by facilitating their degradation and elimination $(1,2)$. Indeed, the overexpression of a single chaperone, such as Hsp70, can supels of polyglutamine toxicity (9). However, increasing the level of expression of a single chaperone has met with mixed success in

\section{Inducing the heat shock response as a therapeutic strategy} press neurodegeneration in Drosophila mod- improving disease phenotypes in mouse models of neurodegenerative disease (11). Therefore, it has been proposed that activating the entire HSR, rather than overexpressing a single chaperone, may prove more successful in remediating toxic protein conformations (12). Specifically, it has been suggested that activation of the HSR via the transcription factor heat shock factor 1 (HSF1), a master regulator of the HSR in eukaryotes, may be beneficial in targeting neurodegenerative disorders (12). Consistent with this proposal, a constitutively active form of HSF1 antagonizes polyglutamine aggregation and extends lifespan in a mouse model of HD (13). Furthermore, HSF1 provides a protective function in mice inoculated with prions: Hsf1-knockout mice succumb to prion disease $20 \%$ faster than control mice (14). Thus, strategies to pharmacologically activate the HSR via HSF1 hold promise for the treatment of a broad spectrum of presently incurable protein-misfolding disorders (1).

Under nonstressful physiological conditions, HSF1 is unable to induce the transcription of genes encoding HSR proteins because it is held in a complex with Hsp90 (Figure 1). Thus, one pharmacological strategy to activate the HSR via HSF1 is to use Hsp90 inhibitors, such as geldanamycin and 17-AAG, that dissociate the Hsp90:HSF1 complex and enable HSF1 to stimulate heat shock gene expression. In fly and mammalian cell models of HD and Parkinson disease, treatment with geldanamycin reduces aggregate load and toxicity $(15,16)$. However, the use of geldanamycin and 17-AAG in mammals is complicated by their toxicity and inability to efficiently cross the blood-brain barrier.

\section{HSP990: a brain-penetrant Hsp90 inhibitor}

The careful and elegant study of Labbadia et al. assesses the utility of a brain-penetrant Hsp90 inhibitor, HSP990, in activating the HSR as a potential therapy in a mouse model of HD (10). Using a well-tolerated chronic dosing regimen, the authors administered 
A Early-stage HD

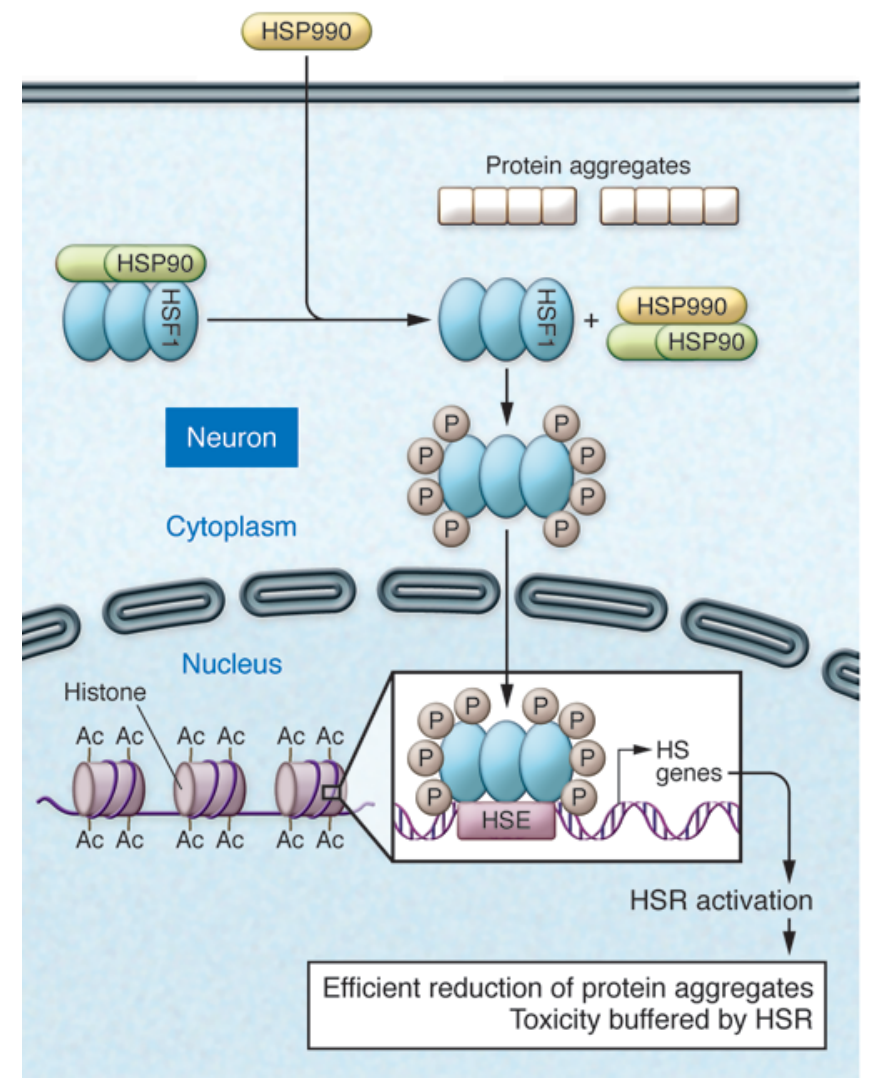

B Late-stage HD

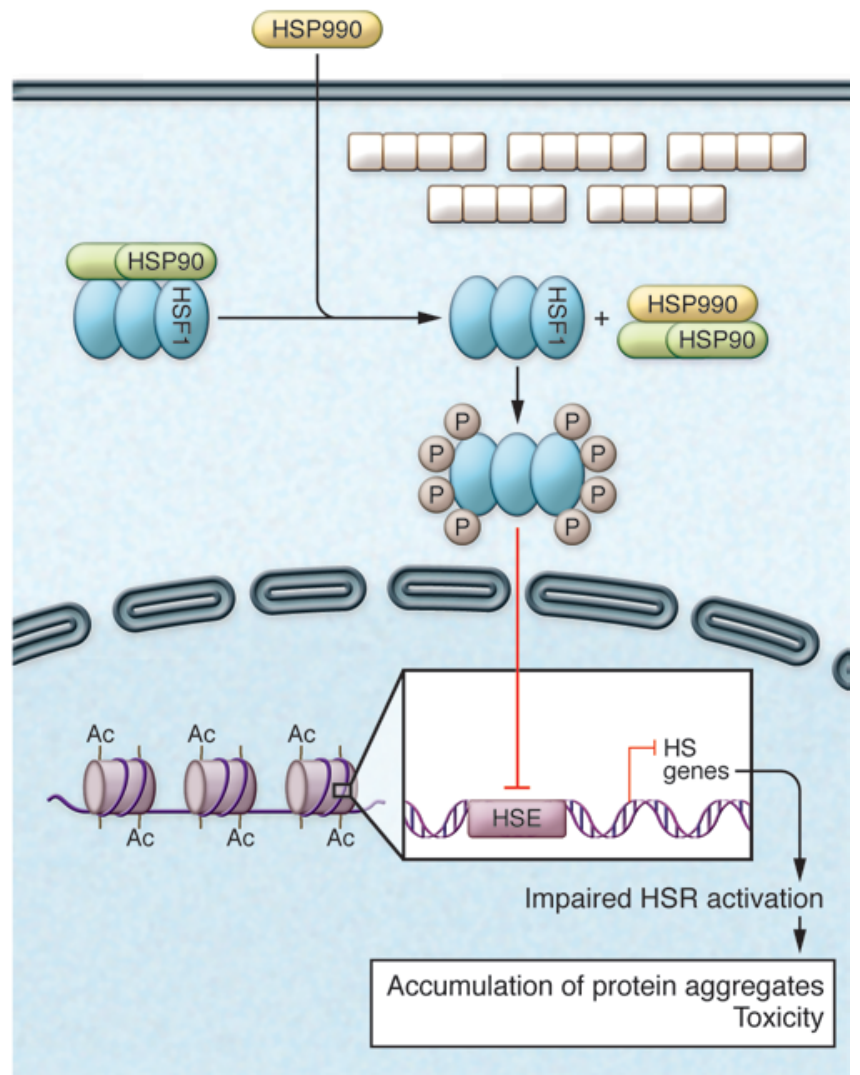

Figure 1

HSR activation varies with disease stage in mice that model HD. Upon inhibition of Hsp90 by the small molecule HSP990, the HSR is activated via HSF1. HSF1 dissociates from its repressive complex with Hsp90, is hyperphosphorylated, and translocates to the nucleus in both early-and latestage HD mice. As HD progresses and the mice age, histone $\mathrm{H} 4$ becomes hypoacetylated at heat shock (HS) gene promoters, preventing efficient transcription of the heat shock genes and impairing the HSR. While early-stage HD mice induce an HSR upon HSP990 treatment and reduce the aggregate burden, late-stage HD mice cannot induce the HSR, and the aggregate burden and toxicity increases. HSE, heat shock element.

HSP990 orally to WT mice and R6/2 transgenic mice, which express exon 1 of a mutant human huntingtin gene with more than 150 CAG repeats. R6/2 mice model HD with an early age of onset and rapid disease progression. Importantly, HSP990 activated the HSR by liberating HSF1 from Hsp90, which in turn led to hyperphosphorylation and activation of HSF1 (Figure 1 and ref. 10). As a consequence, levels of Hsp70, Hsp40, and Hsp25 were greatly increased in the brains of mice. Treatment with HSP990 did not alter the levels of UPR chaperones, indicative of specific induction of the HSR. However, induction of the UPR might also hold value for treating polyglutamine toxicity (17). Promisingly, administration of HSP990 to $\mathrm{R} 6 / 2$ mice resulted in a $20 \%$ decrease in aggregate load in brain tissues and was associated with improved brain weight (Figure 1 and ref. 10). Additionally, a general improvement in disease phenotype was indicated by a 30\% improvement in rotarod performance. However, not all symptoms of HD were ameliorated. HSP990 treatment did not improve body weight, grip strength, or exploratory activity in the R6/2 mice. It remains unclear why only a subset of HD-related phenotypes was partially rescued.

\section{The HSR becomes impaired as disease progresses}

Surprisingly, the benefits afforded by HSP990 were transient. Rotarod performance of R6/2 HD mice improved with treatment at 8 and 10 weeks of age, but not at 14 weeks, which suggests that impairment of the HSR might occur upon HD progression (10). This decline in behavior with HD progression was also reflected in Hsp expression levels. In young mice, HSP990-induced upregulation of Hsp70, Hsp40, and Hsp25 was comparable in WT and R6/2 mice. However, by 8 weeks of age, impairment in Hsp induction was already apparent for the $\mathrm{R} 6 / 2$ mice, and this deterioration continued with age until the mice reached end-state disease at 15 weeks (Figure 1 and ref. 10). In these late-stage disease R6/2 mice, treatment with HSP990 failed to induce Hsp expression. Thus, HSP990 efficacy declined sharply in R6/2 mice in an age-dependent manner. Importantly, this impairment of the HSR was not an artifact of the R6/2 model of HD. The HSR also became impaired in late-stage HdbQ150 knockin mice, which model lateonset HD and express full-length human huntingtin with a polyglutamine tract of 150 residues. Taken together, these data suggest it will be important to determine whether the HSR also becomes impaired in the brains of HD patients.

Further studies revealed that HSR impairment occurred at the level of transcription (10). This impaired transcription could have several potential origins. To 
induce Hsp expression, HSF1 must dissociate from its repressive complex with Hsp90, translocate to the nucleus, and be hyperphosphorylated (Figure 1). Coimmunoprecipitation studies indicated that HSP990 effectively dissociated Hsp90 and HSF1 even in older R6/2 mice (10). Additionally, after HSP990 treatment, HSF1 was hyperphosphorylated and localized to the nucleus in R6/2 brain tissue. Thus, HSF1 is activated by HSP990 equally well in WT and R6/2 mice. However, the authors further noted that alterations in chromatin architecture in older R6/2 mice precluded HSF1 from engaging the promoters of heat shock genes. As the R6/2 mice aged, lower levels of HSF1 binding at various heat shock gene promoters was observed upon HSP990 treatment. Furthermore, chromatin immunoprecipitation using the Hsp70 promoter revealed lower levels of associated RNA polymerase II in R6/2 than in WT brain tissue, consistent with reduced transcription. Additionally, hypoacetylation of histone $\mathrm{H} 4$ was observed at various heat shock genes as disease progressed (10), which might reduce the ability of HSF1 to bind target promoters by reducing chromatin accessibility (Figure 1). However, additional experiments showed that the Hsp70 promoter region was equally accessible in both WT and R6/2 mice. Thus, accessibility per se does not appear to be the issue.

Labbadia et al. propose a model whereby as HD progresses, histone $\mathrm{H} 4$ becomes hypoacetylated at heat shock gene promoters, perhaps because polyglutamine aggregates sequester key histone acetyltransferases (10). Through an undefined mechanism, hypoacetylation precludes HSF1 binding to heat shock gene promoters. Thus, the HSR is impaired (Figure 1). The discovery that the HSR becomes impaired as disease progresses in HD model mice is perhaps the authors' most significant finding. The reduced ability to launch the HSR is likely to exacerbate HD progression by rendering cells incapable of responding appropriately to environmental stressors. However, it remains unknown whether similar defects in the HSR occur in HD patients. Thus, it is possible (although unlikely, in our view) that these deficits reflect events unique to mouse models of HD that are unrelated to events in HD patients. It will be important, although challenging, to corroborate these findings in HD patient tissue or cell lines. Of note, the authors did not generate survival curves (10), so the therapeutic value of HSP990 treatment with regard to longevity remains unknown. However, even a transient improvement of disease phenotype may bring an enhanced quality of life to HD patients, regardless of any potential increase in lifespan.

\section{Prospects for inducing the HSR as an HD therapy}

Overall, the work of Labbadia and colleagues (10) demonstrates that HSF1-mediated activation of the HSR via Hsp90 inhibition may hold great therapeutic value. However, it also emphasizes the complexities of targeting the HSR pathway, because impairment of the HSR was an unforeseen effect of HD progression. This unexpected deterioration of the HSR indicates that simply developing therapeutics to activate this pathway might not be sufficient to remediate HD and perhaps other proteinmisfolding disorders. Indeed, the HSR also becomes impaired in prion-infected cells, although this deficit is rescued by geldanamycin (18). For HD, it may be necessary to combine approaches that activate HSF1 with approaches that circumvent the impairment of the HSR, which might include methods to increase histone H4 acetylation. Moving forward, it will be key to define precisely how HD-induced alterations in chromatin architecture preclude HSF1 binding. More broadly, combination therapies that synergize to target multiple aspects of proteostasis, including direct interactions with the misfolded protein itself, are promising weapons against various protein-misfolding disorders $(8,19)$.

Finally, it should be noted that eliciting the HSR is not the only effect of inhibiting Hsp90. Hsp90 controls the maturation of many key signal transducers, including kinases and transcription factors (20). Consequently, Hsp90 is a potent phenotypic capacitor. Inhibition of Hsp90 exposes cryptic genetic variation that can generate diverse, complex multigenic phenotypes that are difficult to predict, depend on the specific genetic background, and are not necessarily advantageous (20). Indeed, inhibition of Hsp90 can yield highly stable changes in chromatin states that can persist in a heritable manner $(21,22)$. Thus, inhibiting Hsp90 might even accentuate the alterations in chromatin architecture that impair the HSR in mice that model HD. In any case, it is probable that an HSR in which Hsp90 is not inhibited will be a more effective means to restore proteostasis. Thus, small molecules that activate HSF1 without inhibiting Hsp90 could be a critical innovation. One such small molecule, HSF1A, has recently been isolated in a cunning yeast screen (23). HSF1A increased the expression levels of several chaperones and consequently diminished the toxicity of polyglutamine proteins in mammalian cell culture and Drosophila models (23). We suggest that brain-penetrant variants of HSF1A and other potential small molecules that activate HSF1 without inhibiting Hsp90 should also be explored in mouse models of HD.

\section{Summary}

The work of Labbadia et al. (10) has revealed important complications that must be addressed if the HSR response is to be induced as a potential HD therapy. The way is now open to define how the HSR becomes impaired and how this impairment might be prevented or reversed. Of particular interest is the development of small-molecule drugs that might antagonize HSR impairment and synergize with HSP990 to treat mice that model HD. We suggest that specific histone deacetylase (HDAC) inhibitors might be interesting lead candidates to combine with HSP990. In isolation, HDAC inhibitors have exhibited therapeutic effects in mice that model HD (4). Moreover, they could prevent the hypoacetylation of histone $\mathrm{H} 4$ that correlates with HSR impairment (10) and thereby help maintain an active HSR.

\section{Acknowledgments}

The authors acknowledge support from an American Heart Association postdoctoral fellowship (M.E. Jackrel), NIH grants 1DP2OD002177-01 and 5R21NS067354-02 (J. Shorter), an Ellison Medical Foundation New Scholar in Aging Award (J. Shorter), a Bill and Melinda Gates Grand Challenges Explorations Award (J. Shorter), and a grant from the Packard Center for ALS Research at Johns Hopkins (J. Shorter).

Address correspondence to: James Shorter, Department of Biochemistry and Biophysics, Perelman School of Medicine, University of Pennsylvania, 422 Curie Boulevard, Philadelphia, Pennsylvania 19104, USA. Phone: 215.573.4256; Fax: 215.573.7290; E-mail: jshorter@mail.med.upenn.edu.

1. Balch WE, Morimoto RI, Dillin A, Kelly JW. Adapting proteostasis for disease intervention. Science. 2008;319(5865):916-919.

2. Powers ET, Morimoto RI, Dillin A, Kelly JW, Balch WE. Biological and chemical approaches to diseases of proteostasis deficiency. Annu Rev Biochem. 2009;78:959-991.

3. Cushman M, Johnson BS, King OD, Gitler AD, 
Shorter J. Prion-like disorders: blurring the divide between transmissibility and infectivity. J Cell Sci. 2010;123(pt 8):1191-1201.

4. Crook ZR, Housman D. Huntington's disease: can mice lead the way to treatment? Neuron 2011;69(3):423-435.

5. Scherzinger E, et al. Huntingtin-encoded polyglutamine expansions form amyloid-like pro tein aggregates in vitro and in vivo. Cell. 1997 90(3):549-558.

6. Nekooki-Machida Y, Kurosawa M, Nukina N, Ito $\mathrm{K}$, Oda T, Tanaka M. Distinct conformations of in vitro and in vivo amyloids of huntingtin-exon 1 show different cytotoxicity. Proc Natl Acad Sci US A. 2009;106(24):9679-9684.

7. Lo Bianco C, et al. Hsp104 antagonizes alphasynuclein aggregation and reduces dopaminergic degeneration in a rat model of Parkinson disease. J Clin Invest. 2008;118(9):3087-3097.

8. Mu TW, et al. Chemical and biological approaches synergize to ameliorate protein-folding diseases. Cell. 2008;134(5):769-781.

9. Warrick JM, Chan HYE, Gray-Board GL, Chai Y, Paulson HL, Bonini NM. Suppression of polyglutamine-mediated neurodegeneration in Drosophila by the molecular chaperone HSP70. Nat Genet.
1999;23(4):425-428.

10. Labbadia J, et al. Altered chromatin architecture underlies progressive impairment of the heat shock response in mouse models of Huntington disease. J Clin Invest. 2011;121(8):3306-3319.

11. Hay DG, et al. Progressive decrease in chaperone protein levels in a mouse model of Huntington's disease and induction of stress proteins as a therapeutic approach. Hum Mol Gen. 2004; 13(13):1389-1405.

12. Westerheide SD, Morimoto RI. Heat shock response modulators as therapeutic tools for diseases of protein conformation. J Biol Chem. 2005;280(39):33097-33100.

13. Fujimoto $M$, et al. Active HSF1 significantly suppresses polyglutamine aggregate formation in cellular and mouse models. J Biol Chem. 2005 280(41):34908-34916.

14. Steele AD, et al. Heat shock factor 1 regulates lifespan as distinct from disease onset in prion disease. Proc Natl Acad Sci U S A. 2008;105(36):13626-13631.

15. Auluck PK, Bonini NM. Pharmacological prevention of Parkinson disease in Drosophila. Nat Med. 2002;8(11):1185-1186

16. Sittler A, et al. Geldanamycin activates a heat shock response and inhibits huntingtin aggregation in a cell culture model of Huntington's disease. Hum Mol Gen. 2001;10(12):1307-1315.

17. Duennwald ML, Lindquist S. Impaired ERAD and ER stress are early and specific events in polyglutamine toxicity. Genes Dev. 2008;22(23):3308-3319.

18. Winklhofer KF, Reintjes A, Hoener MC, Voellmy $\mathrm{R}$, Tatzelt J. Geldanamycin restores a defective heat shock response in vivo. J Biol Chem. 2001; 276(48):45160-45167.

19. Shorter J. Emergence and natural selection of drugresistant prions. Mol Biosyst. 2010;6(7):1115-1130.

20. Jarosz DF, Taipale M, Lindquist S. Protein homeostasis and the phenotypic manifestation of genetic diversity: principles and mechanisms. Annu Rev Genet. 2010;44:189-216.

21. Sangster TA, Queitsch C, Lindquist S. Hsp90 and chromatin: where is the link? Cell Cycle. 2003; 2(3):166-168

22. Sollars V, Lu X, Xiao L, Wang X, Garfinkel MD, Ruden DM. Evidence for an epigenetic mechanism by which $\mathrm{Hsp} 90$ acts as a capacitor for morphological evolution. Nat Genet. 2003;33(1):70-74.

23. Neef DW, Turski ML, Thiele DJ. Modulation of heat shock transcription factor 1 as a therapeutic target for small molecule intervention in neurodegenerative disease. PLoS Biol. 2010;8(1):e1000291.

\title{
Oxidized CaMKII: a "heart stopper" for the sinus node?
}

\author{
Sabine Huke and Björn C. Knollmann
}

Division of Clinical Pharmacology, Vanderbilt University School of Medicine, Nashville, Tennessee, USA

\begin{abstract}
Each normal heart beat is triggered by an electrical impulse emitted from a group of specialized cardiomyocytes that together form the sinoatrial node (SAN). In this issue of the JCI, Swaminathan and colleagues demonstrate a new molecular mechanism that can disrupt the normal beating of the heart: angiotensin II - typically found in increased levels in heart failure and hypertension - oxidizes and activates $\mathrm{Ca}^{2+} /$ calmodulin-dependent kinase II via NADPH oxidase activation, causing SAN cell death. The loss of SAN cells produces an electrical imbalance termed the "source-sink mismatch," which may contribute to the SAN dysfunction that affects millions of people later in life and complicates a number of heart diseases.
\end{abstract}

The electrical impulses that trigger the heart to beat originate from a group of specialized cardiomyocytes that together form the sinoatrial node (SAN). The SAN is a complex anatomical structure located in the wall of the right atrium, near the entrance to the superior vena cava. The SAN triggers billions of heart beats during an individual's lifetime. Neurohormonal regulation of the SAN allows us to adapt our cardiac output to precisely match life's rapidly changing demands: cardiac out-

Conflict of interest: The authors have declared that no conflict of interest exists.

Citation for this article: J Clin Invest. 2011; 121(8):2975-2977. doi:10.1172/JCI58389. put is reduced during times of rest and increased during physical and emotional exercise. Not surprisingly, SAN dysfunction (SND) affects millions of individuals later in life; it also complicates a number of heart diseases. While a large body of work has elucidated the molecular signaling processes that regulate physiological pacemaking, much less is known about the molecular signaling that causes SND (1). SND is characterized by physiologically inappropriate heart rates, most often sinus bradycardia (a regular but abnormally slow heart rate), and the only currently available treatment option is implantation of an electrical pacemaker. The typical patient is elderly and presents with additional cardiac pathology of an ischemic, inflammatory, or degenerative nature. SND also frequently occurs when an individual develops heart failure. Histological studies demonstrate a loss of SAN cells and increased fibrosis in SAN tissue obtained postmortem (2, 3 ), suggesting that cell death and tissue remodeling importantly contribute to SND. In this issue of the JCI, Swaminathan and coworkers elegantly combine studies in mice and human tissue to demonstrate a molecular chain reaction that can cause SND (4). The study demonstrates a heretofore unrecognized molecular mechanism responsible for SND and provides a clear target for developing new treatments aimed at preventing SND in the future.

\section{Ang II-induced $\mathrm{Ca}^{2+} /$ calmodulin- dependent kinase II oxidation causes SAN cell death and SND}

Anderson and colleagues have shown previously that Ang II induces myocardial dysfunction and heart failure at least in part via myocyte apoptosis (5). They recognized at that time that inhibition of $\mathrm{Ca}^{2+}$ calmodulin-dependent kinase II (CaMKII) provided protection against myocyte cell 